\title{
Anisotropy of small-scale stratospheric irregularities retrieved from scintillations of a double star $\alpha$-Cru observed by GOMOS/ENVISAT
}

\author{
V. Kan ${ }^{1}$, V. F. Sofieva ${ }^{2}$, and F. Dalaudier ${ }^{3}$ \\ ${ }^{1}$ A. M. Obukhov Institute of Atmospheric Physics Russian Academy of Sciences, Moscow, Russia \\ ${ }^{2}$ Earth observation, Finnish Meteorological Institute, Helsinki, Finland \\ ${ }^{3}$ Université Versailles St-Quentin, UPMC University Paris 06, CNRS/INSU, LATMOS-IPSL, 78280 Guyancourt, France
}

Correspondence to: V. F. Sofieva (viktoria.sofieva@fmi.fi)

Received: 3 May 2012 - Published in Atmos. Meas. Tech. Discuss.: 13 July 2012

Revised: 30 September 2012 - Accepted: 16 October 2012 - Published: 14 November 2012

\begin{abstract}
In this paper, we discuss estimating anisotropy of air density irregularities (ratio of characteristic horizontal and vertical scales) from satellite observations of bichromatic scintillations of a double star whose components are not resolved by the detector. The analysis is based on fitting experimental auto- and cross-spectra of scintillations by those computed using the 3-D spectral model of atmospheric irregularities consisting of anisotropic and isotropic components. Application of the developed method to the scintillation measurements of the double star $\alpha$-Cru by GOMOS (Global Ozone Monitoring by Occultation of Stars) fast photometers results in estimates of anisotropy coefficient of $\sim 15-20$ at altitudes $30-38 \mathrm{~km}$, as well as other parameters of atmospheric irregularities. The obtained estimates of the anisotropy coefficient correspond to small-scale irregularities, close to the buoyancy scale.
\end{abstract}

\section{Introduction}

\subsection{Anisotropy of air density irregularities}

Under conditions of stable stratification, the buoyancy force gives special characteristics to the vertical direction. This means that atmospheric stratified flows are anisotropic. Perturbations in the stratified flows, which are usually considered as random and allow the spectral description, are also anisotropic. In the spectral description, the anisotropy of air density irregularities is usually defined as the ratio of characteristic horizontal and vertical scales (e.g. Dalaudier and Gurvich, 1997; Gurvich and Brekhovskikh, 2001;
Sukoriansky and Galperin, 2008). In our paper, we will follow this definition of the anisotropy coefficient.

The irregularities in atmospheric density and temperature fields are generated by internal gravity waves $(\mathrm{GW})$ and turbulence. From the dispersion relation for medium- and lowfrequency gravity waves (e.g. Fritts and Alexander, 2003), the anisotropy can be estimated as $N / \omega(N$ is the BruntVäisälä frequency, $\omega$ is intrinsic frequency of gravity waves). In case of low-frequency GWs with $\omega \sim f$ ( $f$ is the Coriolis parameters), this gives $N / f \sim 100-200$, and these estimates are related to the scales close to the dominant wavelengths, i.e. close to the outer scale of the GW spectrum. Dominant vertical and horizontal wavelengths of the gravity wave field have been experimentally estimated using radiosonde data, rocket soundings and satellite data (Wang et al., 2005; Ern et al., 2004; Alexander et al., 2008; Wang and Alexander, 2010). Despite different assumptions and approximations used in analyses, all these data give large values for anisotropy estimates: $\sim 100-300$ from radiosondes (Wang et al., 2005), 50-300 from satellite measurements by CRISTA (Ern et al., 2004) and HIRLDS (Alexander et al., 2008), 180-1000 from radio occultation measurements by COSMIC (Wang and Alexander, 2010), with a tendency to larger anisotropy in the equatorial region. These observations confirm the abovementioned theoretical predictions of anisotropy at large scales.

The concept of variable anisotropy (Dalaudier and Gurvich, 1997; Gurvich, 1997; Gurvich and Chunchuzov, 2008) assumes reduction of anisotropy towards small scales. The model with variable anisotropy proposed in Gurvich and Chunchuzov (2008) gives different slopes of 1-D vertical 
and horizontal spectra, in accordance with observations. The anisotropy of air density irregularities in the buoyancy subrange is poorly explored experimentally. The only analysis of the angular dependence of the radar cross section of backscattering (Gurvich and Kon, 1993) results in the estimates of anisotropy $\sim 10-15$, for the vertical scales of a few meters at the altitude $\sim 20 \mathrm{~km}$.

\subsection{Using scintillation measurements for studying air density irregularities}

Random irregularities of air density cause fluctuations in light intensity (scintillation), when a star is observed through the Earth atmosphere. Statistical properties of scintillations are defined by the structure of air density irregularities and by properties of the light source. Satellite measurements of stellar scintillation have been used for remote sensing of irregularities in the atmospheres of planets and their satellites (Hubbard et al., 1988; Raynaud et al., 2004) and in the Earth atmosphere (Gurvich and Kan, 2003a,3b; Sofieva et al., 2007a,b, 2009b). The theory of weak scintillation developed by Tatarskii $(1961,1971)$ allows a relatively simple analysis of measurement data.

Based on observations of stellar scintillations on board the MIR station, a model of 3-D wavenumber spectrum of atmospheric irregularities has been developed. This model consists of two components: anisotropic irregularities (the model of saturated gravity waves) and isotropic irregularities (the model of Kolmogorov turbulence) (Gurvich and Brekhovskikh, 2001; Gurvich and Kan, 2003a). The theoretical scintillation auto-spectra for a single star have been analysed in detail in Gurvich and Brekhovskikh (2001), Gurvich and Kan (2003a) and Sofieva et al. (2007a). The methodology for reconstruction of the parameters of 3-D-spectrum of atmospheric irregularities from experimental scintillation auto-spectra has been used successfully for analyses of scintillation data from the MIR station (Gurvich and Kan, 2003a,b) and then (revisited and adapted) for bulk reconstruction of GW and turbulence parameters using scintillation measurements by GOMOS on board Envisat (Gurvich et al., 2007; Sofieva et al., 2007a,b, 2009b). This method allows reconstruction of all parameters of the spectral model except for the anisotropy coefficient of anisotropic irregularities, because scintillation auto-spectra are not sensitive to this parameter provided it is large. Detailed discussion of the methods, their possibilities and limitations is presented in the review (Sofieva et al., 2012).

Simultaneous scintillation measurements by two GOMOS photometers in blue $\left(473-527 \mathrm{~nm}, \lambda_{\mathrm{B}} \approx 500 \mathrm{~nm}\right)$ and red (646-698 nm, $\lambda_{\mathrm{R}} \approx 672 \mathrm{~nm}$ ) wavelengths with the sampling frequency of $1000 \mathrm{~Hz}$ (Bertaux et al., 2010; Kyrölä et al., 2004) allow analyzing also cross-spectra and coherency spectra of bi-chromatic scintillations in addition to scintillation auto-spectra. Theoretical evaluation of cross-spectra and coherency spectra has been discussed in Gurvich et al. (2005), Kan (2004) and Kan et al. (2001). Gurvich et al. (2005) have shown that coherency and correlation can be used for estimating the ratio of anisotropic and isotropic components of scintillations.

\subsection{Scintillations of double stars and the work objective}

The influence of extended light sources on scintillations has been studied in Tatarskii $(1961,1971)$, Cohen et al. (1967) and Little and Hewish (1966). Tatarskii $(1961,1971)$ gave the quantitative explanation of the known fact that planets scintillate less than stars for the same zenith angles. In Little and Hewish (1966) and Cohen et al. (1967), scintillations of extended radio-sources in the interplanetary plasma, including double sources, have been discussed. In these papers, theoretical relations for variance, spectra and correlation function have been obtained for point and extended sources, and large datasets have been analysed to determine the angular size and structure of the sources.

Specific features of single-wavelength scintillations of double stars during occultation in the Earth atmosphere (with its characteristic irregularities) have been considered in Sofieva et al. (2009a) based on exemplary scintillation measurements by GOMOS in occultation of the double star $\alpha$ Cru. The double structure of the star leads to modulation of scintillation spectra and reduced variance of scintillation.

The objective of this paper is to demonstrate additional capabilities of the scintillation method when using cross and coherency scintillation spectra in occultations of a double unresolved star. We propose a method for estimating the anisotropy coefficient in the 3-D spectral model of saturated GW and apply this method to GOMOS observations of the double star $\alpha$-Cru. The paper is organised as follows. Section 2 briefly presents the main approximations used in the analysis. In Sect. 3, we consider the properties of chromatic scintillations of double unresolved stars and the possibility of their use for estimating the parameters of atmospheric irregularities. The measurement results and their comparison with theoretical calculations are presented in Sect. 4. The discussion and summary conclude the paper.

\section{Assumptions and approximations}

For analyses, the following models and approximations are used (Gurvich and Kan, 2003a,b; Sofieva et al., 2007a,b, 2009b):

- two-component model of the 3-D spectrum of atmospheric inhomogeneities;

- phase screen approximation;

- weak scintillation assumption.

The properties of the random medium are described by the 3-D spectrum $\Phi_{v}$ of relative fluctuations of refractivity (or 
density) of air, which consists of two statistically independent components, anisotropic $\Phi_{\mathrm{W}}$ and isotropic $\Phi_{\mathrm{K}}$ :

$\Phi_{\nu}(\kappa)=\Phi_{\mathrm{W}}(\kappa)+\Phi_{\mathrm{K}}(\kappa)$.

The component $\Phi_{\mathrm{W}}$ corresponds to anisotropic irregularities generated by an ensemble of internal gravity waves (Gurvich and Brekhovskikh, 2001; Gurvich and Kan, 2003a; Sofieva et al., 2007a); it has a power spectrum with the slope -5 :

$$
\begin{aligned}
\Phi_{\mathrm{W}}= & C_{\mathrm{W}} \eta^{2}\left(\kappa_{z}^{2}+\eta^{2} \kappa_{\perp}^{2}+K_{z, \mathrm{~W}}^{2}\right)^{-5 / 2}\left(1+\kappa^{2} / \kappa_{z, \mathrm{~W}}^{2}\right)^{-1}, \\
& \kappa^{2}=\eta^{2} \kappa_{\perp}^{2}+\kappa_{z}^{2}, \kappa_{\perp}^{2}=\kappa_{x}^{2}+\kappa_{y}^{2} .
\end{aligned}
$$

This spectrum is a generalisation of a well-known 1-D model of the saturated gravity waves (e.g. Smith et al., 1987) for irregularities having a statistical structure symmetric with respect to the vertical (Gurvich, 1997). The anisotropic spectrum $\Phi_{\mathrm{W}}$ is defined by the following parameters: inner $l_{\mathrm{W}}=2 \pi / \kappa_{z}, \mathrm{~W}$ and outer $L_{\mathrm{W}}=2 \pi / K_{z}, \mathrm{~W}$ vertical scales $\left(\kappa_{z, \mathrm{~W}}\right.$ and $K_{z, \mathrm{~W}}$ are the corresponding characteristic wavenumbers that bounds the part of the spectrum with the slope -5 ), the structure characteristic $C_{\mathrm{W}}$ (intensity of anisotropic fluctuations) and the anisotropy coefficient $\eta$. In this work, we use a simple model with $\eta=$ Const $>1$ (Gurvich and Brekhovskikh, 2001; Sofieva et al., 2007a). The anisotropy coefficient $\eta$ is assumed to be constant for all scales of the anisotropic spectrum $\Phi_{\mathrm{W}}$, but the method used is mostly sensitive to small scales. The corresponding 1-D vertical spectrum has a slope -3 , as the saturated GW spectrum (Smith et al., 1987).

Isotropic component $\Phi_{\mathrm{K}}$ having the slope of the 3D power spectrum $-11 / 3$ corresponds to the Kolmogorov model of locally isotropic turbulence (Monin and Yaglom, 1975):

$$
\begin{aligned}
\Phi_{\mathrm{K}}(\kappa) & =0.033 C_{\mathrm{K}} \kappa^{-11 / 3} \exp \left(-\left(\kappa / \kappa_{\mathrm{K}}\right)^{2}\right), \kappa^{2} \\
& =\kappa_{x}^{2}+\kappa_{y}^{2}+\kappa_{z}^{2} .
\end{aligned}
$$

For scintillations, it is characterised by two parameters: the structure characteristic $C_{\mathrm{K}}$ and the inner scale $l_{\mathrm{K}}=2 \pi / \kappa_{\mathrm{K}}$ (Tatarskii, 1971). Insufficient sampling frequency of GOMOS photometers does not allow retrievals of $l_{\mathrm{K}}$ (Sofieva et al., 2007a), therefore we assume $l_{\mathrm{K}} \ll \rho_{\mathrm{F}}=\sqrt{2 \pi \lambda D} \approx 3.5 \mathrm{~m}$, where $\rho_{\mathrm{F}}$ is the Fresnel scale and $D$ is the distance from ray perigee to a detector (for GOMOS, $D \approx 3225 \mathrm{~km}$ ) for computing theoretical isotropic spectra.

Analysis of the scintillation is greatly simplified by using the phase screen approximation, which assumes that the influence of extended atmosphere is replaced by a phase screen that produces the same phase modulation of the propagated light waves. This screen is a plane placed perpendicular to the incident rays and passing through the Earth centre. The use of the phase screen approximation is justified by the fact that the effective length of interaction of stellar light and the atmosphere, which is a few hundreds of kilometers, is small compared to the distance $D$ from the phase screen to observer. Intensity fluctuations at the exit from the atmosphere are negligibly small and they are formed during the propagation of light waves from the phase screen to the detector. The properties of the phase screen and conditions of its applicability to satellite measurements are discussed in detail in, for example, Gurvich (1984), Gurvich and Brekhovskikh (2001) and Dalaudier et al. (2001). The effect of changing aspect angles of anisotropic irregularities along the ray is taken into account in computation of the theoretical scintillation spectra (Gurvich, 1984; Gurvich and Brekhovskikh, 2001).

Assumption of weak scintillation (scintillation variance is much smaller than unity; Tatarskii, 1961, 1971) provides a fairly simple relations between the 3-D spectrum of atmospheric irregularities and the measured 1-D scintillation spectrum (Gurvich and Brekhovskikh, 2001; Gurvich and Kan, 2003a; Sofieva et al., 2007a). The regime of weak scintillations for low-orbit satellites is realised for the ray perigee altitudes above $30 \mathrm{~km}$ (Gurvich and Kan, 2003a,b; Sofieva et al., 2007a). It is important to note that for model (1), spectra of weak scintillations can also be considered as a sum of statistically independent spectral components corresponding to anisotropic and isotropic irregularities. The frequency scintillation spectra can be transformed into spatial wavenumber spectra using the hypothesis of "frozen" field of irregularities, which is valid due to the large velocity of the line of sight. For strong scintillations (ray perigee altitudes less than $30 \mathrm{~km}$ ), the consideration becomes much more complicated. Properties of strong scintillation spectra in occultation experiments and some possibilities for retrievals of the information about the structure of atmospheric irregularities are discussed, in particular, in Gurvich et al. (2011).

\section{A qualitative analysis - method of estimating atmospheric parameters}

Figure 1 shows schematically the geometry of occultation measurements. The left panels shows the plane containing blue and red rays for one component of a double star and the satellite. The right panel shows the phase screen, the plane perpendicular to light rays, with the trajectories of red and blue rays of a double star that are recorded simultaneously by the detector. Due to dispersion of regular refraction, light rays of different colours have different trajectories in the atmosphere, which are shifted vertically with respect to each other (rays 1 and 3 and 2 and 4 corresponding to the components of the double star). This chromatic shift $\Delta_{c}$ depends on wavelengths and on ray perigee altitude. For GOMOS photometers, $\Delta_{\mathrm{c}} \approx 5.5 \mathrm{~m}$ at $34 \mathrm{~km}$ (Dalaudier et al., 2001). Note that vertical scales and velocities in the phase screen differ from those at the observation plane by the factor $q$, the refractive attenuation (Dalaudier et al., 2001). Since $q \geq 0.9$ above $30 \mathrm{~km}$, we set $q=1$ in the present qualitative analysis. 

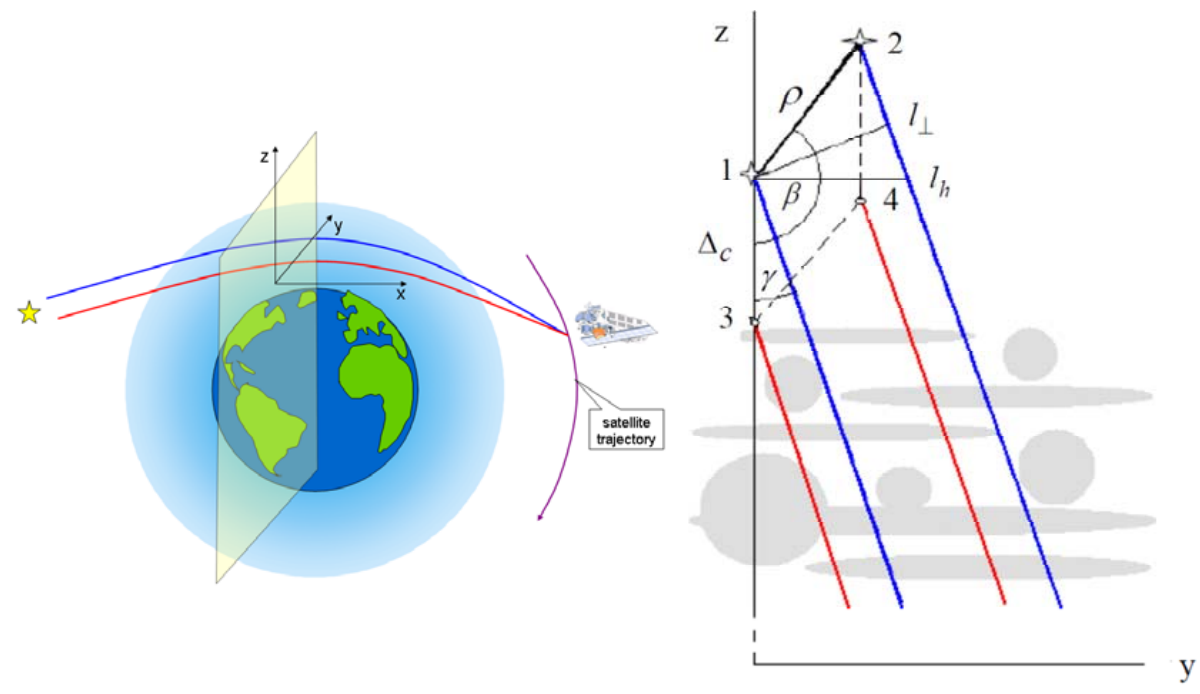

Fig. 1. Left panel: illustration of occultation geometry. Yellow plane represents the phase screen. Blue and red lines denote coloured ray trajectories for a single star, which are recorded simultaneously by the instrument. Right panel: a sketch of ray trajectories of binaries in the phase screen during occultations. The points $1-4$, which are located in a vertices of a parallelogram, are the points of intersection of light rays with the phase screen (1-2 for the blue photometer and 3-4 for the red photometer). $\Delta_{c}$ is the chromatic shift, i.e. the vertical distance between red and blue rays (1-3 for the first component of a double star, 2-4 are for the second component). Atmospheric irregularities are schematically shown by grey circles and ellipses. Anisotropic irregularities are oblate spheroids with horizontal isotropy and small axis along the vertical.

In case of a single star, the observation geometry is determined by the obliquity angle $\gamma$ (the angle between the ray trajectory in the phase screen and the local vertical) (Gurvich and Brekhovskikh, 2001; Gurvich and Kan, 2003a). In the considered GOMOS observations of $\alpha$-Cru, $-19^{\circ} \leq \gamma \leq 36^{\circ}$ with an annual cycle.

In case of a double star, there are two additional trajectories of blue and red rays (2 and 4 in Fig. 1) of the second component of star, which are parallel to the trajectories of the first component. The additional parameters that define geometry of double star observations are angular $\theta$ or spatial $\rho=\theta D$ separation of binaries and the angle $\beta$ between $\bar{\rho}$ and the local vertical (orientation of binaries) (Sofieva et al., 2009a). According to the coordinates of components $\alpha-\mathrm{Cru}_{\mathrm{A}}$ and $\alpha-\mathrm{Cru}_{\mathrm{B}}$ presented in The Hipparcos and Tycho Catalogues (ESA, 1997); Tycho Double Star Catalogue: http://vizier.u-strasbg.fr/viz-bin/VizieR, the separation of binaries in 2002-2003 was $\theta \approx 4.0$ arcsec and $\rho \approx 62.6 \mathrm{~m}$. The angle $\beta$ has an annual cycle because of the precession of Envisat orbit. The minimal distance between the ray trajectories of the same color corresponding to binaries is $l_{\perp}=\rho|\sin (\gamma-\beta)|$ and their horizontal separation is $l_{\mathrm{h}}=L_{\perp} / \cos \gamma$ (see Fig. 1). Thus for a double star, two GOMOS photometers allow probing the atmosphere along the four spaced paths. While the chromatic refraction separates the rays of different colors along the vertical, the separation of trajectories of binaries can be in any direction. Changing geometry of observations due to the precession of the
ENVISAT orbit can provide a separation of binaries trajectories from zero to a maximum value (Sofieva et al., 2009a).

If the rays from binaries pass successively through the same irregularities, then the signal in each photometer is the sum of the same (accurate within amplitudes) but shifted in time random realisations. If this time shift is larger than the inverse frequency band of scintillations (inverse Nyquist frequency), then the spectral density of shifted realisations will be cosine modulated with the maximal modulation coefficient (contrast) (Sofieva et al., 2009a):

$C_{\max }=\frac{2 I_{01} I_{02}}{I_{01}^{2}+I_{02}^{2}}$,

where $I_{01}$ and $I_{02}$ are vacuum intensities of binaries. For $\alpha$ Cru, which has binaries of similar intensities, $C_{\max } \approx 0.9$. In this regard, $\alpha$-Cru is of greatest interest among all occultations of double stars available in GOMOS database.

For large-scale anisotropic irregularities, the modulation of scintillation spectra is close to maximal even in case of moderate anisotropy $\eta$. However, for small scales (high frequencies), the modulation can be significantly reduced in case of moderate anisotropy and/or sufficiently large separations $l_{\mathrm{h}}$ that exceed the characteristic horizontal scale of irregularities $\eta / \kappa_{z}$. This modulation reduction can be used for estimating the anisotropy of small-scale irregularities. The modulation frequency is defined by the vertical velocity $v_{\mathrm{v}}$ of the satellite and by the vertical separation of binaries $\rho|\cos \beta|$ (Sofieva et al., 2009a):

$f_{\mathrm{m}, \mathrm{a}}=v_{\mathrm{v}} /(\rho|\cos \beta|)$. 
Under our assumptions, the scintillation spectra corresponding to isotropic irregularities are defined by the Fresnel scale $\rho_{\mathrm{F}}$ in the whole frequency range (Tatarskii, 1971). The modulation of scintillation spectra corresponding to isotropic irregularities is observed when

$\rho_{\mathrm{F}}>2 \pi l_{\perp}=2 \pi \rho|\sin (\gamma-\beta)|$.

Because $\rho \gg \rho_{\mathrm{F}}$ for $\alpha$-Cru, this condition is satisfied only for very small range of angles, $|\gamma-\beta| \leq 1^{\circ}-2^{\circ}$ and we will not consider such occultations in the present analysis.

Coherency of bi-chromatic scintillations has been discussed in Kan et al. (2001), Kan (2004) and Gurvich et al. (2005). For GOMOS occultations of $\alpha$-Cru (available for $|\gamma| \leq 36^{\circ}$ ), coherency of anisotropic scintillation above $30 \mathrm{~km}$ (where the weak scintillation assumption is valid) is close to maximal. Isotropic scintillations are non-coherent below $40 \mathrm{~km}$ for $|\gamma| \geq 15^{\circ}$ (and at higher altitudes for larger $\gamma)$. Therefore, the measured cross-spectrum is related only to anisotropic scintillations, in the altitude range $\sim 30-40 \mathrm{~km}$ for $|\gamma| \geq 15^{\circ}$. (Note that the condition $|\gamma|>15^{\circ}$ is satisfied for about half of all GOMOS observations of $\alpha$-Cru).

We use the coherency of anisotropic scintillations and non-coherency of isotropic scintillations of $\alpha$-Cru at altitudes 30-40 km (for $|\gamma|>15^{\circ}$ ) for separating anisotropic $V_{\mathrm{a}}$ and isotropic $V_{\mathrm{i}}$ components in the measured (total) scintillation spectra $V=V_{\mathrm{a}}+V_{\mathrm{i}}$. By subtracting the autospectrum of isotropic scintillations (in the adopted approximation, it is defined by the single parameter, the turbulent structure characteristic $V_{\mathrm{i}}=V_{\mathrm{i}}\left(C_{\mathrm{K}}\right)$ ) from experimental auto-spectra, we get the anisotropic component of scintillation auto-spectra. We perform fitting of air density irregularities parameters in two steps. First, the estimate of $C_{\mathrm{K}}$ is obtained by fitting the coherency spectrum, $\operatorname{coh}_{\mathrm{a}}(f)=\frac{\left|V_{\mathrm{a}}\left(f, \lambda_{\mathrm{B}}, \lambda_{\mathrm{R}}\right)\right|^{2}}{V_{\mathrm{a}}\left(f, \lambda_{\mathrm{B}}\right) V_{\mathrm{a}}\left(f, \lambda_{\mathrm{R}}\right)}$ (where $V_{\mathrm{a}}\left(f, \lambda_{\mathrm{B}}, \lambda_{\mathrm{R}}\right)$ is the experimental cross-spectrum of bi-chromatic scintillations, $V_{\mathrm{a}}\left(f, \lambda_{\mathrm{B}}\right)=V\left(\begin{array}{ll}f, & \lambda_{\mathrm{B}}\end{array}\right)-V_{\mathrm{i}}^{\bmod }\left(\begin{array}{lll}f, & \lambda_{\mathrm{B}}, & \left.C_{\mathrm{K}}\right) \text { and }\end{array}\right.$ $V_{\mathrm{a}}\left(f, \lambda_{\mathrm{R}}\right)=V\left(f, \lambda_{\mathrm{R}}\right)-V_{\mathrm{i}}{ }^{\bmod }\left(f, \lambda_{\mathrm{R}}, C_{\mathrm{K}}\right)$ are the experimental auto-spectra of anisotropic scintillations) to the theoretical value $\sim 1$ in the whole range of the analysed frequencies. Here $V_{\mathrm{i}}$ mod $\left(f, \lambda, C_{\mathrm{K}}\right)$ stays for the modeled autospectrum of isotopic scintillations. This constitutes the first step of our retrieval; it gives the estimates of the turbulent structure characteristic $C_{\mathrm{K}}$ and the spectrum of anisotropic scintillations $\hat{V}_{\mathrm{a}}$. Then, at the second step, the detected auto spectra $\hat{V}_{\mathrm{a}}\left(f, \lambda_{\mathrm{R}}\right), \hat{V}_{\mathrm{a}}\left(f, \lambda_{\mathrm{B}}\right)$ of anisotropic scintillations and the cross-spectrum $V_{\mathrm{a}}\left(f, \lambda_{\mathrm{B}}, \lambda_{\mathrm{R}}\right)$ are used for fitting the parameters of the anisotropic component $C_{\mathrm{W}}, L_{\mathrm{W}}, l_{\mathrm{W}}$ and $\eta$. In this paper, for demonstrating the capabilities of the method and obtaining initial estimates of the parameters of atmospheric inhomogeneities, we have applied visual fitting. The reconstruction of the parameters can be also formulated in the form of non-linear optimization analogous to Sofieva et al. (2007a), if needed. The retrieval process is illustrated below.

\section{Experimental data and retrieval results}

The information about occultations of $\alpha$-Cru chosen for the analysis is presented in Table 1. For clarity, we selected occultations where the frequency modulation of the scintillation spectra is approximately the same: $f_{\mathrm{m}, \mathrm{a}} \approx 50-60 \mathrm{~Hz}$. To analyse the modulation, the observations with sufficiently separated trajectories are preferred. Therefore, we used occultation with $l_{\mathrm{h}}$ from $70 \mathrm{~m}$ (this is close to the maximal separation for $\alpha$-Cru) down to $30 \mathrm{~m}$. Furthermore, the occultations with high values of the coherency spectrum were selected, because the uncertainty of cross- and coherency spectra is inversely proportional not only to the number of averaged periodograms but also to the values of coherency spectrum (Bendat and Piersol, 1986).

In the selected occultations, the values of the coherency spectrum (smoothed over modulations) are not less than $0.25-0.3$ for frequencies up to $350 \mathrm{~Hz}$. This frequency range was used for analyses (the Nyquist frequency is $500 \mathrm{~Hz}$ ). The angle $\beta$ characterizing the orientation of binaries was estimated from the known vertical velocity of the satellite and the measured modulation frequency, according to Eq. (1). The experimental values of $\beta$ agree with the theoretical ballistic model that uses the parameters of the Envisat orbit and the coordinates of the $\alpha$-Cru components.

We have used experimental data at altitudes $30-38 \mathrm{~km}$, where scintillations are pronounced and, at the same time, satisfy the weak scintillation assumption (as seen in Table 1, the relative scintillation variance does not exceed 0.25 ). First, samples corresponding to the vertical length of $2 \mathrm{~km}$ were used. The chromatic shift was compensated by the shifting of the sample. The periodograms were averaged with a window of variable width: at low frequencies with window of constant quality $f / \Delta f=2$ and with the window of the constant width $\Delta f_{\mathrm{c}} \approx 12 \mathrm{~Hz}$ at higher frequencies, in order to resolve the modulation of frequency $f_{\mathrm{m}, \mathrm{a}} \geq 50 \mathrm{~Hz}$. Then the estimates of auto-spectra and the components of the crossspectra were averaged over the whole considered altitude range $\Delta h=30-38 \mathrm{~km}$. The total number of independent periodograms in the spectral window $\Delta f_{\mathrm{c}}$ was 36 . The scintillation spectra were corrected for instrumental noise, which was estimated using the measurements above the atmosphere.

Figure 2 shows exemplary experimental scintillation spectra at two orbits, which are characterised by enhanced coherency (separation of trajectories $l_{\mathrm{h}}$ is twice larger for the orbit 04728 than for 05051). Modulation of the scintillation auto-spectra is due to the fact that the 2-D fields of relative intensity fluctuations at the satellite plane corresponding to each component of the double star are identical and only shifted by $\rho$ with respect to each other (Sofieva et al., 2009a). The modulation depth depends on characteristic scintillation scales and the shift of scintillation fields in the corresponding directions. In the considered altitude range, only anisotropic component of scintillations is modulated, while the isotropic component is not modulated. The 
Table 1. Information about the selected occultations of $\alpha$-Cru.

\begin{tabular}{|c|c|c|c|c|c|c|c|c|c|c|c|}
\hline Date and orbit & Ray perigee & $\begin{array}{l}\text { Scint rms, } \\
\text { blue/red \% }\end{array}$ & $\begin{array}{l}\text { Obliquity } \\
\gamma, \text { deg }\end{array}$ & $\begin{array}{l}\text { Modulation } \\
\text { freq. } \\
f_{\mathrm{m}, \mathrm{a}}, \mathrm{Hz}\end{array}$ & $\begin{array}{r}\text { Orientation } \\
\beta, \operatorname{deg}\end{array}$ & $\begin{array}{c}\text { Horiz. } \\
\text { Separ. } \\
l_{\mathrm{h}}, \mathrm{m}\end{array}$ & $\begin{array}{l}C_{\mathrm{K}} \cdot 10^{9} \\
\mathrm{~m}^{-2 / 3}\end{array}$ & $\begin{array}{r}C_{\mathrm{W}} \cdot 10^{11} \\
\mathrm{~m}^{-2}\end{array}$ & $\begin{array}{l}L_{\mathrm{W}} \\
\mathrm{km}\end{array}$ & $\underset{\mathrm{m}}{l_{\mathrm{W}}}$ & $\begin{array}{c}\text { Anisotropy } \\
\eta\end{array}$ \\
\hline $\begin{array}{l}3 \text { Jan } 2003 \\
\# 04418\end{array}$ & $7.2^{\circ} \mathrm{S}, 69.1^{\circ} \mathrm{E}$ & $21 / 23$ & 30.8 & 64 & -38.0 & 68 & 0.57 & 4.0 & 1.5 & 16 & 20 \\
\hline $\begin{array}{l}3 \text { Jan } 2003 \\
\# 04419\end{array}$ & $7.2^{\circ} \mathrm{S}, 43.9^{\circ} \mathrm{E}$ & $20 / 22$ & 30.8 & 64 & -38.0 & 68 & 0.53 & 4.0 & & 19 & 20 \\
\hline $\begin{array}{l}21 \text { Jan } 2003 \\
\# 04675\end{array}$ & $1.4^{\circ} \mathrm{N}, 95.6^{\circ} \mathrm{E}$ & $37 / 41$ & 35.0 & 53 & -20.2 & 63 & 3.2 & 9.5 & 2.5 & 10 & 16 \\
\hline $\begin{array}{l}21 \text { Jan } 2003 \\
\# 04677\end{array}$ & $1.5^{\circ} \mathrm{N}, 45.3^{\circ} \mathrm{E}$ & $35 / 38$ & 35.0 & 53 & -20.2 & 63 & 2.2 & 12.6 & 2.0 & 16 & 16 \\
\hline $\begin{array}{l}25 \text { Jan } 2003 \\
\# 04727\end{array}$ & $3.2^{\circ} \mathrm{N}, 132.4^{\circ} \mathrm{W}$ & $29 / 32$ & 35.2 & 52 & -13.5 & 58 & 2.2 & 6.4 & 2.5 & 15 & 18 \\
\hline $\begin{array}{l}25 \mathrm{Jan} 2003 \\
\# 04728\end{array}$ & $3.2^{\circ} \mathrm{N}, 157.6^{\circ} \mathrm{W}$ & $33 / 37$ & 35.3 & 52 & -13.5 & 58 & 2.5 & 8.4 & & 11 & 17 \\
\hline $\begin{array}{l}31 \text { Jan } 2003 \\
\# 04810\end{array}$ & $5.8^{\circ} \mathrm{N}, 60.5^{\circ} \mathrm{W}$ & $43 / 47$ & 35.2 & 50 & -8.0 & 51 & 3.8 & 16.0 & 2.0 & 14 & 16 \\
\hline $\begin{array}{l}31 \text { Jan } 2003 \\
\# 04822\end{array}$ & $6.2^{\circ} \mathrm{N}, 2.3^{\circ} \mathrm{W}$ & $43 / 48$ & 35.2 & 50 & -7.5 & 51 & 4.8 & 20.0 & 2.5 & 14 & 13 \\
\hline $\begin{array}{l}10 \text { Feb } 2003 \\
\# 04964\end{array}$ & $10.5^{\circ} \mathrm{N}, 334.8^{\circ} \mathrm{W}$ & $28 / 31$ & 33.8 & 50 & 2.0 & 40 & 1.0 & 9.2 & 1.5 & 15 & 15 \\
\hline $\begin{array}{l}10 \text { Feb } 2003 \\
\# 04965\end{array}$ & $10.4^{\circ} \mathrm{N}, 0.0^{\circ} \mathrm{E}$ & $27 / 30$ & 33.8 & 50 & 2.5 & 39 & 0.70 & 12.1 & 1.5 & 25 & 20 \\
\hline $\begin{array}{l}11 \text { Feb } 2003 \\
\# 04975\end{array}$ & $10.7^{\circ} \mathrm{N}, 108.4^{\circ} \mathrm{E}$ & $29 / 33$ & 33.6 & 50 & 3.5 & 38 & 2.0 & 10.0 & 2.0 & 11 & 13 \\
\hline $\begin{array}{l}11 \mathrm{Feb} 2003 \\
\# 04976\end{array}$ & $10.7^{\circ} \mathrm{N}, 83.3^{\circ} \mathrm{E}$ & $21 / 22$ & 33.6 & 50 & 3.5 & 38 & 0.73 & 5.4 & & 16 & 14 \\
\hline $\begin{array}{l}15 \text { Feb } 2003 \\
\# 05045\end{array}$ & $12.6^{\circ} \mathrm{N}, 147.2^{\circ} \mathrm{E}$ & $35 / 40$ & 32.3 & 50.5 & 7.6 & 31 & 2.3 & 10.0 & 2.5 & 11 & 17 \\
\hline $\begin{array}{l}15 \text { Feb } 2003 \\
\# 05047\end{array}$ & $12.6^{\circ} \mathrm{N}, 96.9^{\circ} \mathrm{E}$ & $29 / 32$ & 32.3 & 50.5 & 7.6 & 31 & 1.6 & 7.7 & 2.5 & 12 & 14 \\
\hline $\begin{array}{l}15 \text { Feb } 2003 \\
\# 05051\end{array}$ & $12.6^{\circ} \mathrm{N}, 3.7^{\circ} \mathrm{W}$ & $23 / 24$ & 32.2 & 50.5 & 7.5 & 30 & 0.45 & 5.6 & 1.6 & 18 & 19 \\
\hline
\end{tabular}

cross-spectrum contains only anisotropic (coherent) component. As observed in Fig. 2, moduli of cross-spectra have a deeper modulation than auto-spectra. This is due to the presence of isotropic (non-modulated) component in autospectra, in contrast to cross-spectra. The modulation of coherency spectra is defined by the ratio of the scintillation components: anisotropic (fully coherent, with a modulated auto-spectrum) and isotropic (non-coherent, with a nonmodulated auto-spectrum). At maxima, the anisotropic component prevails, while the isotropic component is significant at minima. The systematic reduction of coherency with frequency is related to reduction of the anisotropic scintillation share at high frequencies (scales less than $l_{\mathrm{W}}$ ).

Figure 3 illustrates detecting anisotropic and isotropic components of scintillation auto-spectra in the frequency range below $350 \mathrm{~Hz}$. The values of the structure characteristic $C_{\mathrm{K}}$ were obtained by fitting the coherency spectra of anisotropic scintillations to the theoretical value close to 1 : grey lines in the right panels show the original measured coherency spectra, while black lines show the coherency of the anisotropic component (black dashed lines show the theoretical anisotropic coherency spectra). The retrieved estimates of $C_{\mathrm{K}}$ for the selected dataset are presented in Table 1.

Left and centre panels show detected auto-spectra of anisotropic and isotropic scintillations, and right panels show moduli of cross-spectra and coherency spectra of the anisotropic component.

The detected auto-spectra and the measured modulus of cross-spectra of anisotropic scintillations are used then for estimating the parameters of the 3-D spectrum of atmospheric irregularities $\Phi_{\mathrm{W}}$. The fitting parameters are the structure characteristic $C_{\mathrm{W}}$, outer and inner scales $L_{\mathrm{W}}$ and $l_{\mathrm{W}}$, and the anisotropy coefficient $\eta: C_{\mathrm{W}}$ is defined by the amplitude of the spectra, $L_{\mathrm{W}}$, and $l_{\mathrm{W}}-$ by the characteristic limiting frequencies, $\eta$ - by the modulation of anisotropic spectra. In the occultation 05051, spectra have a larger modulation depth in high-frequency domain $f \geq 150 \mathrm{~Hz}$, compared to that in 04728 . This is due to the twice larger horizontal separation of binaries trajectories in 04728 (while the anisotropy coefficients are similar in these two occultations). The estimates of anisotropy coefficient correspond to small 

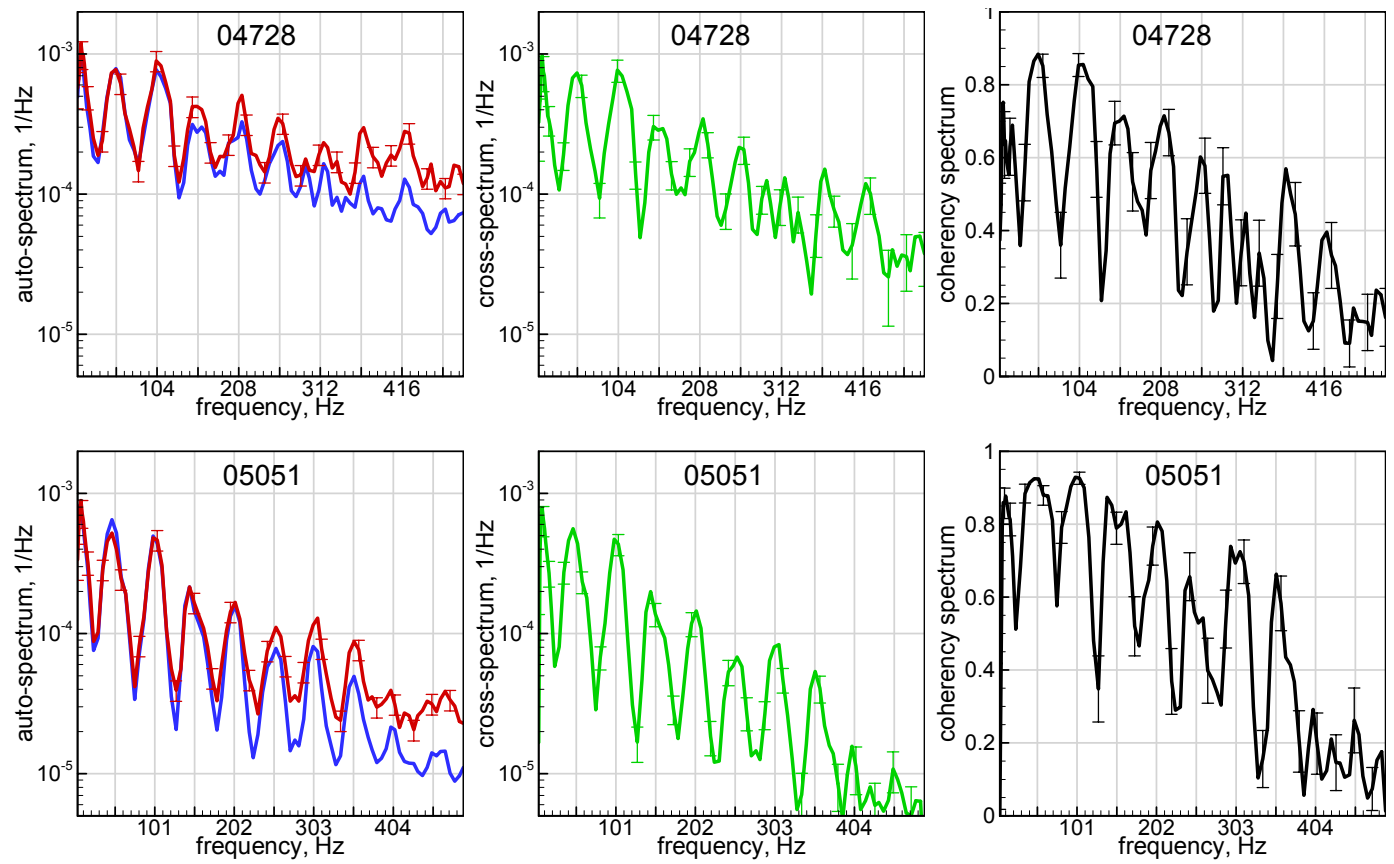

Fig. 2. Experimental scintillation spectra of $\alpha$-Cru. Left panels: auto-spectra (line colours correspond to wavelengths of fast photometers), centre panels: moduli of cross-spectra, right panels: coherency spectra. Vertical grid hereafter shows the modulation periods. Error bars show rms uncertainty of spectra estimates (Bendat and Piersol, 1986) (rms uncertainties for red and blue auto-spectra are similar).
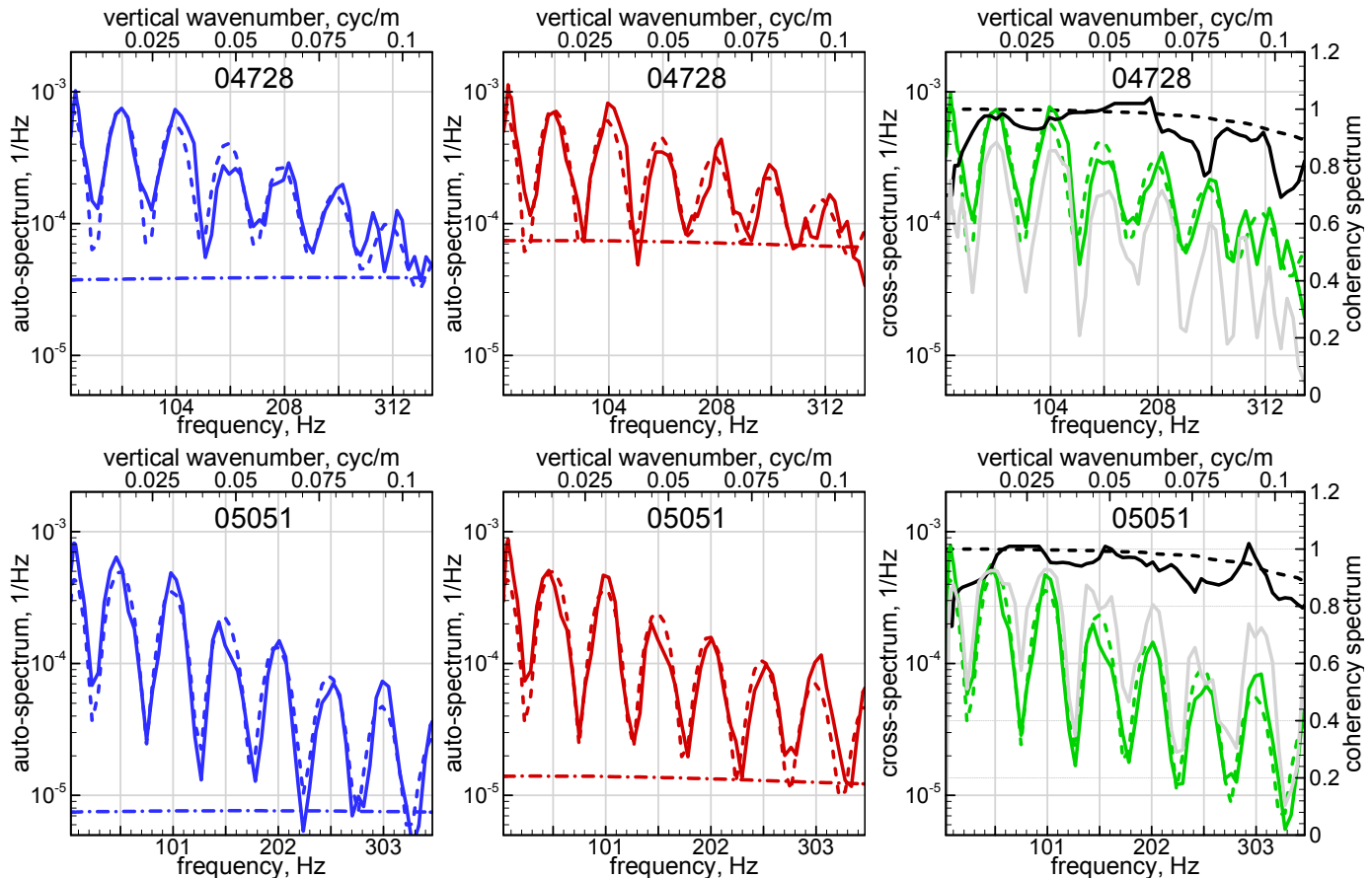

Fig. 3. Illustration of detecting isotropic and anisotropic components of scintillation spectra. Left panels (for the blue photometer) and centre panels (for the red photometer): detected experimental (solid lines) and theoretical (dashed lines) auto-spectra of anisotropic scintillations and theoretical auto-spectra of isotropic scintillations (dot-dashed lines). Right panels: experimental (solid lines) and theoretical (dashed lines) moduli of cross-spectra (green) and coherency spectra (black) of the detected anisotropic component; grey lines indicate the measured coherency spectrum. 
scales close to $l_{\mathrm{W}}$ : in the considered occultations, of vertical scales $10-20 \mathrm{~m}$, as seen in Fig. 3.

The retrieved parameters $C_{\mathrm{W}}, L_{\mathrm{W}}, l_{\mathrm{W}}$ and $\eta$ for the selected occultations are presented in Table 1. Missing $L_{\mathrm{W}}$ estimates for some occultations in Table 1 are because the outer scale is not detected in these occultations (a reduction of spectra amplitude at large scales). In computing scintillation spectra, we have taken into account the effects related to bandwidth of the optical filters and to the finite integration time (1 ms) of the GOMOS instrument (Kan et al., 2001). The aliasing effect (Bendat and Piersol, 1986) has also been taken into account. In particular, some reduction of the theoretical coherency spectrum from 1 at high frequencies (dashed lines in Fig. 3) is due to aliasing.

The estimates of the parameters of irregularities presented in Table 1 exhibit rather large variations, which exceeds significantly uncertainty of the estimated parameters. This feature noticed in earlier works; it is related to the natural variability (intermittency) of the parameters of gravity waves and turbulence in the atmosphere (Gurvich and Kan, 2003a; Sofieva et al., 2007a). In general, the estimated structure characteristics $C_{\mathrm{K}}$ and $C_{\mathrm{W}}$, the inner $l_{\mathrm{W}}$ and outer $L_{\mathrm{W}}$ scales of anisotropic irregularities are in good agreement with the previous results of the reconstruction of parameters from scintillation data (Gurvich and Kan, 2003a,b; Sofieva et al., 2007a), especially if one takes into account that $\eta=30$ has been set in these previous retrievals. As can be seen in Table 1, anisotropic inhomogeneities are characterised by a rather strong anisotropy $\eta=15-20$ for the small scales comparable with the GW inner scale.

\section{Summary and discussion}

In this paper, we have demonstrated how bi-chromatic scintillation of double unresolved stars can be used for studying the structure of air density irregularities. Specific features of double star scintillation (the modulation of autoand cross-spectra) allow estimation of the anisotropy coefficient $\eta$ of the 3-D spectral model of anisotropic air density irregularities, in addition to other parameters of $\mathrm{GW}$ and turbulence spectra retrieved in previous studies from the monochromatic scintillations of single stars (Gurvich and Kan, 2003a; Sofieva et al., 2007a). Our analysis is based on fitting the parameters of the 3-D spectral model of air density irregularities to the experimental auto- and cross-spectra of scintillations.

The main limitation in using chromatic scintillations is a larger uncertainty of experimental estimates of cross- and coherency spectra (compared to that of auto-spectra). Therefore, their use is possible only for scintillation measurements with high coherency or/and with large averaging of periodograms. In the considered case, the periodogram averaging is limited by the frequency window $\Delta f_{\mathrm{c}}$ and the altitude interval $\Delta h$. Therefore, we have selected occultations with enhanced coherency and have used a limited frequency range in the estimated spectra. Thus, our parameter estimates correspond to cases where the anisotropic component is prevailing over the isotropic one in the 3-D spectrum of atmospheric irregularities.

In our work, the use of a simple model with the constant anisotropy $\eta=$ Const is based on the fact that the modulation of the $\alpha$-Cru scintillation spectra gives information about the anisotropy for small-scale irregularities with the vertical scales $\leq 20 \mathrm{~m}$. Irregularities of larger scales provide the maximal modulation for $\eta=$ Const, especially if $\eta$ increases with increasing scales as assumed in the variable anisotropy models.

The presented method uses only a limited altitude range $30-40 \mathrm{~km}$, which is needed for the proposed two-step retrievals in order to ensure non-coherency of isotropic scintillations. This sequencing makes the data analysis transparent and useful for demonstrating the capability of the method. The proposed method can be further improved with one-step inversion of the parameters of the two-component spectral model of air density irregularities. This might allow the retrievals of the anisotropy coefficient also at higher altitudes, where the isotropic bi-chromatic scintillations are partially correlated. The feasibility study for such retrievals will be the subject of future work.

The method of detecting anisotropic and isotropic components by using the coherency spectra (the first step of the proposed retrieval method) can be applied also to the occultations of single stars, in addition to the method described in Gurvich and Kan (2003a) and Sofieva et al. (2007a).

Our analysis has shown that anisotropic inhomogeneities are characterised by a rather large anisotropy $\eta=15-20$ for the small scales comparable with the inner GW scale. The previous estimate $\eta \geq 25$ presented in Alexandrov et al. (1990) is a qualitative estimate. The estimates of anisotropy at small scales are of high interest, because this range corresponds to the transition of the saturated GW spectrum to the turbulent spectrum. However, the anisotropy at small scales is practically unexplored experimentally for the considered altitude range. The estimates $\eta=10-15$ at vertical scales of a few meters obtained using MST radar measurements (Gurvich and Kon, 1993) correspond to lower altitudes, $\sim 20 \mathrm{~km}$, and smaller scales.

The other measurements at such small scale are very scarce in the considered altitude range $30-40 \mathrm{~km}$. Another source of information about anisotropy of small-scale atmospheric irregularities is quasi-horizontal observations of single stars (in such occultations, $\gamma \approx 90^{\circ}$ at the minimal ray perigee altitude). The method for estimating the anisotropy coefficient by using auto-spectra and coherency spectra in such occultations is discussed in Kan (2004). However, there are only a few such occultations in the whole GOMOS dataset.

The previous analyses and theoretical estimates reviewed in the introduction show that the anisotropy is large, 
$\sim 100-300$ at large scales close to the dominant wavelengths. Our analysis has shown that the irregularities with relatively small vertical scales $\sim 10-20 \mathrm{~m}$ still preserve sufficiently strong anisotropy $\sim 15-20$. This can be interpreted as reduction of anisotropy towards small scales, and thus can serve as an experimental argument in favor of the variable anisotropy models.

Acknowledgements. The authors thank A. S. Gurvich and V. V. Vorobiev for useful discussions and comments. The authors thank ESA, ACRI-ST and the GOMOS team for the GOMOS data. The work of V. Kan was supported by Russian Foundation for Basic Research, grant 12-05-00335. The work of V. Sofieva was supported by the Academy of Finland (MIDAT and ASTREX projects).

Edited by: M. Weber

\section{References}

Alexander, M. J., Gille, J., Cavanaugh, C., Coffey, M., Craig, C., Eden, T., Francis, G., Halvorson, C., Hannigan, J., Khosravi, R., Kinnison, D., Lee, H., Massie, S., Nardi, B., Barnett, J., Hepplewhite, C., Lambert, A., and Dean, V.: Global estimates of gravity wave momentum flux from high resolution dynamics limb sounder (HIRDLS) observations, J. Geophys. Res., 113, D15S18, doi:10.1029/2007JD008807, 2008.

Alexandrov, A. P., Grechko, G. M., Gurvich, A. S., Kan, V., and Savchenko, S. A.: Temperature spectra in the stratosphere from observations of star scintillations from space, Izv. Acad. Sci. USSR Atmos. Ocean. Phys., 26, 1-8, 1990.

Bendat, J. S. and Piersol, A. G.: Random Data: Analysis and Measurement Procedures, 2nd Edn., J. Wiley, New York, 1986.

Bertaux, J. L., Kyrölä, E., Fussen, D., Hauchecorne, A., Dalaudier, F., Sofieva, V., Tamminen, J., Vanhellemont, F., Fanton d'Andon, O., Barrot, G., Mangin, A., Blanot, L., Lebrun, J. C., Pérot, K., Fehr, T., Saavedra, L., Leppelmeier, G. W., and Fraisse, R.: Global ozone monitoring by occultation of stars: an overview of GOMOS measurements on ENVISAT, Atmos. Chem. Phys., 10, 12091-12148, doi:10.5194/acp-10-12091-2010, 2010.

Cohen, M.-H., Gundermann, E.-J., and Harris, D.-E.: New Limits on the Diameters of Radio Sources, Astrophys. J., 150, p. 767, doi:10.1086/149380, 1967.

Dalaudier, F. and Gurvich, A. S.: A scalar three-dimensional spectral model with variable anisotropy, J. Geophys. Res., 102, 19449-19459, doi:10.1029/97JD00962, 1997.

Dalaudier, F., Kan, V., and Gurvich, A. S.: Chromatic refraction with global ozone monitoring by occultation of stars, I. Description and scintillation correction, Appl. Optics, 40, 866-877, 2001.

Ern, M., Preusse, P., Alexander, M. J., and Warner, C. D.: Absolute values of gravity wave momentum flux derived from satellite data, J. Geophys. Res., 109, D20103, doi:10.1029/2004JD004752, 2004.

Fritts, D. C. and Alexander, M. J.: Gravity wave dynamics and effects in the middle atmosphere, Rev. Geophys., 41, 1003, doi:10.1029/2001RG000106, 2003.
Gurvich, A. S.: Fluctuations in the observations of extraterrestrial cosmic sources through the earth's atmosphere, Radiophys. Quant. Electron., 27, 665-672, 1984.

Gurvich, A. S.: A heuristic model of three-dimensional spectra of temperature inhomogeneities in the stably stratified atmosphere, Ann. Geophys., 15, 856-869, doi:10.1007/s00585-997-0856-x, 1997.

Gurvich, A. S. and Brekhovskikh, V.: A study of turbulence and inner waves in the stratosphere based on the observations of stellar scintillations from space: A model of scintillation spectra, Wave. Random Media, 11, 163-181, 2001.

Gurvich, A. S. and Chunchuzov, I. P.: Model of the ThreeDimensional Spectrum of Anisotropic Temperature Irregularities in a Stably Stratified Atmosphere, Izv. Atmos. Ocean. Phys., 44, 567-582, doi:10.1134/S0001433808050034, 2008.

Gurvich, A. S. and Kan, V.: Structure of air density irregularities in the stratosphere from spacecraft observations of stellar scintillation: 1. Three-dimensional spectrum model and recovery of its parameters, Izv. Atmos. Ocean. Phys., 39, 300-310, 2003 a.

Gurvich, A. S. and Kan, V.: Structure of air density irregularities in the stratosphere from spacecraft observations of stellar scintillation: 2. Characteristic scales, structure characteristics, and kinetic energy dissipation, Izv. Atmos. Ocean. Phys., 39, 311-321, $2003 b$.

Gurvich, A. S. and Kon, A. I.: Aspect sensitivity of radar returns from anisotropic turbulent irregularities, J. Elect. Wave. Appl., 7, 1343-1353, 1993.

Gurvich, A. S., Dalaudier, F., and Sofieva, V. F.: Study of stratospheric air density irregularities based on two-wavelength observation of stellar scintillation by Global Ozone Monitoring by Occultation of Stars (GOMOS) on Envisat, J. Geophys. Res., 110, D11110, doi:10.1029/2004JD005536, 2005.

Gurvich, A. S., Sofieva, V. F., and Dalaudier, F.: Global distribution of CT2 at altitudes 30-50 km from space-borne observations of stellar scintillation, Geophys. Res. Lett., 34, L24813, doi:10.1029/2007GL031134, 2007.

Gurvich, A. S., Vorob'ev, V. V., and Fedorova, O. V.: Strong Scintillation Spectra behind the Atmosphere with Large- and SmallScale Inhomogeneities, Atmos. Ocean. Optics, 24, 347-357, 2011.

Hubbard, W. B., Lellouch, E., and Sicardy, B.: Structure of Scintillations in Neptune's Occultation Shadow, Astron. J., 325, 490502, 1988.

Kan, V.: Coherence and correlation of chromatic stellar scintillations in a space-borne occultation experiment, Izvestia, Atmos. Ocean. Optics, 17, 725-735, 2004.

Kan, V., Dalaudier, F., and Gurvich, A. S.: Chromatic refraction with global ozone monitoring by occultation of stars, II. Statistical properties of scintillations, Appl. Optics, 40, 878-889, 2001.

Kyrölä, E., Tamminen, J., Leppelmeier, G. W., Sofieva, V. F., Hassinen, S., Bertaux, J.-L., Hauchecorne, A., Dalaudier, F., Cot, C., Korablev, O., Fanton D’Andon, O., Barrot, G., Mangin, A., Theodore, B., Guirlet, M., Etanchaud, F., Snoij, P., Koopman, R., Saavedra, L., Fraisse, R., Fussen, D., and Vanhellemont, F: GOMOS on Envisat: An overview, Adv. Space Res., 33, 1020-1028, 2004.

Little, L.-T. and Hewish, A.: Interplanetary scintillation and its relation to the angular structure of radio sources, Mon. Notice. Roy. Astronom. Soc., 134, 221-237, 1966. 
Monin, A. S. and Yaglom, A. M.: Statistical Fluid Mechanics, Volume 2, MIT Press, Cambridge, Massachusetts, 1975.

Raynaud, E., Matcheva, K., Drossart, P., Roques, F., and Sicardy, B.: A re-analysis of the 1971 Beta Scorpii occultation by Jupiter: study of temperature fluctuations and detection of wave activity, Icarus, 168, 324-335, doi:10.1016/j.icarus.2003.10.021, 2004.

Smith, S. A., Fritts, D. C., and VanZandt, T. E.: Evidence of a saturation spectrum of atmospheric gravity waves, J. Atmos. Sci., 44, 1404-1410, 1987.

Sofieva, V. F., Gurvich, A. S., Dalaudier, F., and Kan, V.: Reconstruction of internal gravity wave and turbulence parameters in the stratosphere using GOMOS scintillation measurements, J. Geophys. Res., 112, D12113, doi:10.1029/2006JD007483, 2007a.

Sofieva, V. F., Kyröolá, E., Hassinen, S., Backman, L., Tamminen, J., Seppälä, A., Thölix, L., Gurvich, A. S., Kan, V., Dalaudier, F., Hauchecorne, A., Bertaux, J.-L., Fussen, D., Vanhellemont, F., Fanton D'Andon, O., Barrot, G., Mangin, A., Guirlet, M., Fehr, T., Snoeij, P., Saavedra, L., Koopman, R., and Fraisse, R.: Global analysis of scintillation variance: Indication of gravity wave breaking in the polar winter upper stratosphere, Geophys. Res. Lett., 34, L03812, doi:10.1029/2006GL028132, 2007b.

Sofieva, V. F., Dalaudier, F., Kan, V., and Gurvich, A. S.: Technical note: Scintillations of the double star $\alpha$ Cru observed by GOMOS/Envisat, Atmos. Chem. Phys., 9, 8967-8973, doi:10.5194/acp-9-8967-2009, 2009a.
Sofieva, V. F., Gurvich, A. S., and Dalaudier, F.: Gravity wave spectra parameters in 2003 retrieved from stellar scintillation measurements by GOMOS, Geophys. Res. Lett., 36, L05811, doi:10.1029/2008GL036726, 2009b.

Sofieva, V. F., Dalaudier, F., and Vernin, J.: Review: using stellar scintillation for studies of turbulence in the Earth atmosphere, in: Turbulent Mixing and Beyond Theme Issue of the Philosophical Transactions of the Royal Society, 2012.

Sukoriansky, S. and Galperin, B.: Anisotropic turbulence and internal waves in stably stratified flows (QNSE theory), Physica Scripta, T132, 014036, doi:10.1088/00318949/2008/T132/014036, 2008.

Tatarskii, V. I.: Wave propagation in a turbulent medium, edited by: Silverman, R. A., McGraw-Hill, New York, 1961.

Tatarskii, V. I.: The Effects of the Turbulent Atmosphere on Wave Propagation, Israel Program for Scientific Translations, Jerusalem, 1971.

Wang, L. and Alexander, M. J.: Global estimates of gravity wave parameters from GPS radio occultation temperature data, J. Geophys. Res., 115, D21122, doi:10.1029/2010JD013860, 2010.

Wang, L., Geller, M. A., and Alexander, M. J.: Spatial and temporal variations of gravity wave parameters, Part I: intrinsic frequency, wavelength, and vertical propagation direction, J. Atmos. Sci., 62, 125-142, 2005. 